\title{
ANALYTIC FUNCTIONS WITH PRESCRIBED CLUSTER SETS
}

\author{
L. W. BRINN
}

\begin{abstract}
Suppose that $0<R \leq+\infty$. A monotonic boundary path (mb-path) in $|z|<R$ is a simple continuous curve $z=z(s), 0 \leq s<1$, in $|z|<R$ such that $|z(s)| \rightarrow R$ strictly monotonically as $s \rightarrow 1$. Suppose that $f$ is a complex valued function, defined in $|z|<R$, and that $t$ is any mb-path in $|z|<R$. The cluster set of $f$ on $t$ is the set of those points $w$ on the Riemann sphere for which there exists a sequence $\left\{z_{n}\right\}$ of points of $t$ with $\lim _{n \rightarrow \infty}\left|z_{n}\right|=R$ and $\lim _{n \rightarrow \infty} f\left(z_{n}\right)=w$. The cluster set is denoted by $C_{t}(f)$. If the cluster set is a single point set, that point is called the asymptotic value of $f$ on $t$. If the function $f$ is continuous, then $C_{t}(f)$ is a continuum on the Riemann sphere.

It is a conjecture of $\mathrm{F}$. Bagemihl and $\mathrm{W}$. Seidel that if $\tau$ is a family of mb-paths in $|z|<R$ satisfying certain conditions, and if $\mathcal{K}$ is an analytic set of continua on the Riemann sphere, then there exists a function $f$, analytic in $|z|<R$, such that $\left\{C_{t}(f) \mid t \in \tau\right\}=\mathcal{K}$. A restricted form of the conjecture is mentioned in $[\mathbf{3}$, p. 100].

Our principal results show the correctness of the conjecture in the case that $K$ is the collection of all continua on the Riemann sphere and $T$ is a tress of a certain type. The results are generalized in several directions. In particular, our technique for constructing the analytic function $f$ extends immediately to the case in which $K$ is any closed set of continua on the sphere. Specific examples of closed sets lead to several corollaries.
\end{abstract}

1. Historical background. The construction of analytic functions with prescribed cluster sets along mb-paths has a long history. For instance, in $1933 \mathrm{~S}$. Kierst and E. Szpilrajn constructed a function $f$, analytic in $|z|<1$, which has the extended plane as its cluster set along every radius [8, p. 277].

In the 1950's F. Bagemihl and W. Seidel generalized earlier results of A. Roth [12] to develop a construction technique based on polynomial approximation. They utilized the technique in a series of closely related papers. The results in $[\mathbf{5}]$ involved countable families of mb-paths and were based on the approximation theorems of J. L. Walsh. In [4] Bagemihl and Seidel defined a tress and used the approximation theorem of S. N. Mergelyan to extend their construction technique to this type of (possibly uncountable) path family. Further results were obtained in [3]. Their application of the approximation theorems is both ingenious and somewhat complicated. Later it became possible to simplify proofs by a direct application of Arakeljan's theorem on tangential approximation (see $[\mathbf{1}]$ and $[\mathbf{7}]$ ).

In particular, Bagemihl and Seidel have shown that if $K$ is the collection of all locally connected continua on the Riemann sphere, and if $\tau$ is a tress of a certain type, there is a function $f$, analytic in $|z|<R$, for which $\left\{C_{t}(f) \mid t \in \tau\right\}=\mathcal{K}[\mathbf{3}$, p. 102].

Received by the editors December 11, 1984.

1980 Mathematics Subject Classification (1985 Revision). Primary 30D40, 30E10. 
2. Outline. Our principal result is the construction of a function $f$, analytic in $|z|<R$, with the following property: if $K$ is any continuum on the Riemann sphere, then there are $2^{\aleph_{0}}$ mb-paths in $|z|<R$ on which the cluster set of $f$ is exactly $K$. The paths belong to a tress of a certain type, and convergence to the cluster set is uniform on paths of the tress in a sense to be defined.

We begin by developing the topological notions to be used throughout the paper. We then construct a continuous function with the desired boundary behavior. Finally, we use the approximation theorem of Arakeljan to construct the analytic function $f$. Considerable control over the form of the mb-paths is possible: In particular, they may be required to lie in some central angle, to all end at some point on $|z|=R$, to all be spirals, or even radii. The result is generalized in several directions. Finally, we note that similar (but weaker) results may be obtained using only older approximation theorems of J. L. Walsh. Substantial control over the form of the mb-paths is retained. The paths may not, however, be required to be radii. The polynomial approximation technique is more complicated.

\section{Topological background.}

3.1. DEFINITION. By an $R$-sequence $\left\{q_{i}\right\}$ we mean a sequence $\left\{q_{i}\right\}, i=0,1, \ldots$, such that $0<q_{0}<q_{1}<\cdots$ and $\lim _{i \rightarrow \infty} q_{i}=R$. If $\left\{q_{i}\right\}$ is an $R$-sequence, we denote the circle $|z|=q_{i}$ by $C_{i}$. We denote the closed annulus $q_{i-1} \leq|z| \leq q_{i}$ by $A_{i}$ and a half open annulus $A_{i} \cup A_{i+1} \cup \cdots$ by $B_{i}$.

3.2. Definition. By a subcontinuum of a space $S$ we mean a nonempty compact connected subset of $S$.

We adopt the point of view described in $[\mathbf{3}]$ and consider the subcontinua of the Riemann sphere as points of a suitable space. Let $\rho$ denote the chordal metric on the Riemann sphere $X$. Let $2^{X}$ denote the space of nonempty closed subsets of $X$, and let $d$ denote the Hausdorff metric on $2^{X}$ (see [9, pp. 214-215]). That is, if $P$ and $Q$ are elements of $2^{X}$,

$$
d(P, Q)=\max \left(\sup _{p \in P}\left(\inf _{q \in Q} \rho(p, q)\right), \sup _{q \in Q}\left(\inf _{p \in P} \rho(p, q)\right)\right) .
$$

With the Hausdorff metric, $2^{X}$ is a compact metric space $[\mathbf{1 0}$, pp. 45-47]. Let $\mathcal{L}$ denote the subspace of all subcontinua of $X$. Then $\mathcal{L}$ is closed in $2^{X}[\mathbf{1 0}, \mathrm{p} .139]$ and is thus a compact metric space with metric $d$.

If $p$ and $q$ are points of $X$, then clearly $\rho(p, q)=d(\{p\},\{q\})$. Thus, the mapping $p \rightarrow\{p\}$ embeds $X$ in $2^{X}$. To simplify notation, let us denote $d(\{p\},\{q\})$ by $d(p, q)$. Then the metric $d$ gives the ordinary Euclidean topology on $X$.

Suppose that $T$ is a mb-path in $|z|<R$, that $\left\{q_{i}\right\}$ is an $R$-sequence, and that $f$ is a continuous function, defined in $|z|<R$. Then $C_{t}(f)=\bigcap_{i=1}^{\infty} \overline{f\left(t \cap B_{i}\right)}$. Since the function $f$ is continuous, each of the sets $\overline{f\left(t \cap B_{i}\right)}$ is a continuum in the space $\mathcal{L}$ [6, pp. 108-109]. Also, $C_{t}(f)$ is a continuum in $\mathcal{L}$, and, in the topology of $\mathcal{L}$, $\lim _{i \rightarrow \infty} \overline{f\left(t \cap B_{i}\right)}=C_{t}(f)[\mathbf{9}$, p. 339]; [10, p. 49].

We seek to construct an analytic function $f$ with the boundary behavior described in the previous section. The construction will proceed in several steps. First we will select certain locally connected continua $L_{i j}$ in the space $\mathcal{L}$, and on each continuum $L_{i j}$ we will select certain points $p_{i j k}$. We will then use these continua and associated points to define a continuous finite valued function $g$ whose boundary behavior will determine that of the function $f$. The key to the definition 
of the function $g$ is the Hahn-Mazurkiewicz-Sierpinski theorem. The requirement that $g$ be finite valued introduces certain complications. Finally, we will use polynomial approximation theorems to construct the analytic function $f$.

3.3. Definition. By a finite continuum on the Riemann sphere we mean a continuum which does not contain the point $\infty$.

\subsection{LEMMA. The finite locally connected continua are dense in the space $\mathcal{L}$.}

PROOF. It is known that the locally connected continua are dense in $\mathcal{L}$ (see $[\mathbf{9}$, p. $339 ; \mathbf{1 0}$, pp. 49, 260]). We need only show that, given any locally connected continuum $L$ and any $\varepsilon>0$, there is some finite locally connected continuum $F$ with $d(F, L)<\varepsilon$. If $L$ is finite, let $F=L$. If $L=\{\infty\}$, there is clearly a finite point $p$ with $d(p, \infty)<\varepsilon$. Let $F=\{p\}$. If $L$ contains $\{\infty\}$ as a proper subset, let $p$ be a finite point of $L$, and let $d(p, \infty)=\delta$. Let $\eta=\min (\delta, \varepsilon / 2)$, and let $R=\{z \mid$ $d(z, \infty) \geq \eta\}$. By the Hahn-Mazurkiewicz-Sierpinski Theorem $[10$, p. 256] there exists a continuous function $g$ mapping the unit interval $I$ onto $L$. We may assume that $g(0)=g(1)=p$. Let $G=g^{-1}(R \cap L)$. Then $G$ is a closed nonempty subset of $I$, and $g$ restricted to $G$ is continuous. Each component of $I-G$ is an open interval $(a, b)$ whose endpoints lie in $G$. Moreover, $d(g(a), \infty)=d(g(b), \infty)=\eta$. Thus $g$ has a continuous extension $h: I \rightarrow R$ such that $d(h(x), \infty)=\eta$ if $x \in I-G$. The image $h(I)$ is a finite locally connected continuum $[\mathbf{1 0}$, p. 256] and it is straightforward to verify that if we let $F=h(I)$, then $d(F, L)<\varepsilon$.

Thus, in each case, there is a finite locally connected continuum $F$ with $d(F, L)<$ $\varepsilon$. This completes the proof.

3.5. LEMMA. For each positive integer $i$, there exist finitely many locally connected continua $L_{i j}\left(1 \leq j \leq m_{i}\right)$ and on each continuum $L_{i j}$ there exist finitely many points $p_{i j k}\left(1 \leq k \leq r_{i j}\right)$ with the following properties:

1. Given any continuum $K \in \mathcal{L}$, there are at least two continua $L_{i j_{1}}$ and $L_{i j_{2}}$ $\left(j_{1} \neq j_{2}\right)$ with $d\left(K, L_{i j_{1}}\right)<1 / 2^{i+1}$ and $d\left(K, L_{i j_{2}}\right)<1 / 2^{i+1}$.

2. Given any point $p$ of $l_{i j}$ there exists some point $p_{i j k}$ with $d\left(p, p_{i j k}\right)<1 / 2^{i+1}$.

PROOF. The existence of the continua $L_{i j}$ follows directly from the previous lemma and the fact that the space $\mathcal{L}$ is compact. The requirement in property 1 that $j_{1} \neq j_{2}$ does not imply that $L_{i j_{1}}$ and $L_{i j_{2}}$ must be distinct as point sets. The existence of the points $p_{i j k}$ follows directly from the fact that each continuum $L_{i j}$ is a compact subset of the Riemann sphere.

3.6. Definition. For each positive integer $i$, we will call the continua $L_{i j}$ $\left(1 \leq j \leq m_{i}\right)$ which have the properties of the previous lemma the continua of level $i$. For each continuum $L_{i j}$, we will refer to the points $p_{i j k}\left(1 \leq k \leq r_{i j}\right)$ as the bridge points on $L_{i j}$.

As $i \rightarrow \infty$, the continua of level $i$ become "more nearly dense" in the space $\mathcal{L}$, and simultaneously the bridge points $p_{i j k}$ become "more nearly dense" on each $L_{i j}$.

3.7. LEMMA. If $p$ and $q$ are finite points on the Riemann sphere $X$, then there is a path $t$ from $p$ to $q$ with the following properties:

1. All points on $t$ are finite.

2. If $d(p, q)<\varepsilon$ then $d(z, q)<\varepsilon$ for all points $z$ on $t$.

PROOF. Let $t$ be the shortest great circle arc from $p$ to $q$, interrupted (if necessary) by a sufficiently small circular arc around the point $\infty$. 
3.8. Definition. By a direct path from $p$ to $q$ we mean a path having the properties of Lemma 3.7 .

In the following, to simplify notation we will consider sequences of the form $\left\{\left(L_{i}, p_{i}\right)\right\}$ where $L_{i}$ is a continuum of level $i$ and $p_{i}$ is a bridge point on $L_{i}$ for each $i$. Two pairs $\left(L_{i}, p_{i}\right)$ and $\left(C_{i}, q_{i}\right)$ are to be considered equal if and only if $L_{i}$ and $C_{i}$ are the same indexed continuum $L_{i j}\left(1 \leq j \leq m_{i}\right)$ of level $i$, and $p_{i}$ and $q_{i}$ are the same indexed bridge point $p_{i j k}\left(1 \leq k \leq r_{i j}\right)$ on the continuum $L_{i j}$.

In order to insure uniform convergence to the cluster set, we are interested in sequences of the form $\left\{\left(L_{i}, p_{i}\right)\right\}$ which converge at a predetermined rate. This is the motivation behind the following definition.

3.9. Definition. A sequence $\left\{\left(L_{i}, p_{i}\right)\right\}, i=1,2, \ldots$, is called a sequence of the form $*$ if and only if it satisfies the following conditions for each $i$ :

1. $L_{i}$ is one of the continua $L_{i j}\left(1 \leq j \leq m_{i}\right)$ of level $i$.

2. The point $p_{i}$ is a bridge point $p_{i j k}\left(1 \leq k \leq r_{i j}\right)$ on $L_{i j}$.

3. $d\left(L_{i}, L_{i+1}\right)<1 / 2^{i}$.

4. $d\left(p_{i}, p_{i+1}\right)<1 / 2^{i-1}$.

3.10. Lemma. Suppose that $\left\{\left(L_{i}, p_{i}\right)\right\}$ is a sequence of the form $*$. Then

1. $\lim _{i \rightarrow \infty} L_{i}$ exists and is a continuum $K$ in the space $\mathcal{L}$.

2. $\lim _{i \rightarrow \infty} p_{i}$ exists and is a point $p$ on $K$.

3. For each $i, d\left(L_{i}, K\right) \leq 1 / 2^{i-1}$.

4. For each $i, d\left(p_{i}, p\right) \leq 1 / 2^{i-2}$.

PROOF. It follows directly from condition 3 of Definition 3.9 that the sequence $\left\{L_{i}\right\}$ is a Cauchy sequence in the space $\mathcal{L}$. Since $\mathcal{L}$ is a compact metric space, $\lim _{i \rightarrow \infty} L_{i}$ exists and is a continuum $K \in \mathcal{L}$. Similarly, the sequence $\left\{p_{i}\right\}$ is a Cauchy sequence on the Riemann sphere $X$ and converges to some point $p$ of $X$. Since $p_{i} \in L_{i}$ for each $i$, we must have $p \in K[\mathbf{9}$, p. 335; 10, p. 49]. Properties 3 and 4 are straightforward consequences of the corresponding conditions in Definition 3.9.

3.11. LEMMA. Suppose that $K$ is a continuum of $\mathcal{L}$ and that $p$ is a point of $K$. Then there exist $2^{\aleph_{0}}$ sequences $\left\{\left(L_{i}, p_{i}\right)\right\}$ of the form * with $\lim _{i \rightarrow \infty} L_{i}=K$ and $\lim _{i \rightarrow \infty} p_{i}=p$.

PROOF. For each $i$, it follows from Lemma 3.5 that there are at least two continua $L_{i}$ of level $i$ with $d\left(L_{i}, K\right)<1 / 2^{i+1}$. If $d\left(L_{i}, K\right)<1 / 2^{i+1}$, it follows directly from the definition of the Hausdorff metric and the compactness of $L_{i}$ that there exists a point $q$ of $L_{i}$ with $d(p, q)<1 / 2^{i+1}$. But then there exists a bridge point $p_{i}$ on $L_{i}$ with $d\left(p_{i}, p\right)<1 / 2^{i}$, again by Lemma 3.5. Thus, there are $2^{\aleph_{0}}$ sequences of the form $\left\{\left(L_{i}, p_{i}\right)\right\}$ with $d\left(L_{i}, K\right)<1 / 2^{i+1}$ and $d\left(p_{i}, p\right)<1 / 2^{i}$ for each $i$. These sequences satisfy the requirements of the lemma.

In order to state the basic result we need one additional definition.

3.12. Definition. Let $R, 0<R \leq+\infty$, be given, and let $\left\{q_{i}\right\}$ be an $R$ sequence. Let $\tau$ be a family of mb-paths in $|z|<R$, and let $f$ be a continuous function defined on all paths of the family. We say that convergence to the cluster set is uniform for paths of the family $\tau$ if and only if, given any $\varepsilon>0$, there is some integer $n=n(\varepsilon)$ with the following property: If $i \geq n$ and if $t$ is any mb-path of the family $\tau$, then $d\left(\overline{f\left(t \cap B_{i}\right)}, C_{t}(f)\right)<\varepsilon$.

The notion of uniform convergence to the cluster set can be found in [2]. Tresses are defined in $[\mathbf{4}]$. 


\section{The basic result:}

4.1. THEOREM. Let $R, 0<R \leq+\infty$, be given, and let $\left\{q_{i}\right\}$ be an $R$-sequence. Suppose that $E$ is a perfect, nowhere dense set on the circle $|\tau|=1$ and that $\tau$ is a tress in $|z|<R$ with respect to $E$ such that it is possible to take $E_{n}=E$ for all $n$ in the definition of a tress. Then there exists a function $f$, analytic in $|z|<R$, with the following properties:

1. If $K$ is any continuum on the Riemann sphere, there exist $2^{\aleph_{0}}$ mb-paths of $\tau$ on which the cluster set of $f$ is exactly $K$.

2. Convergence to the cluster set is uniform for paths of $\tau$.

We will construct the analytic function $f$ in several steps. First, we will construct a continuous function $G(x, \tau)$ on $\left[q_{0}, R\right) \times E$ so that, for each continuum $K \in \mathcal{L}$, there are $2^{\aleph_{0}}$ points $\tau \in E$ for which the cluster set of $G(x, \tau)$ as $x \rightarrow R$ is $K$. We will then use the function $G$ to construct a continuous function $g(z)$ on the paths of $\tau$. Finally, we will apply Arakeljan's theorem to construct the required analytic function $f$ in $|z|<R$.

If $a$ and $b$ are points on the circle $|\tau|=1,(a, b)([a, b])$ will denote the nondegenerate open (closed) arc extending from $a$ counterclockwise to $b$.

Since the set $E$ of Theorem 4.1 is closed and nowhere dense on $|\tau|=1$, there exist points $a, b \in E, a \neq b$, with $E \subseteq[a, b]$. Since $a, b \in E,[a, b]-E$ is an open subset of $|\tau|=1$. Let $\left\{\left(a_{n}, b_{n}\right)\right\}, n=1,2, \ldots$, be the components of $[a, b]-E$. Then, the $\left(a_{n}, b_{n}\right)$ are pairwise disjoint open arcs whose endpoints lie in $E$. Moreover, the collection $\left\{\left(a_{n}, b_{n}\right)\right\}$ is infinite (otherwise, $E$ would contain either a nondegenerate arc or an isolated point). We next write $E$ as the intersection of a descending sequence of closed sets $E_{i}$, where each $E_{i}$ is the union of "sufficiently many" disjoint nondegenerate closed arcs on $|\tau|=1$.

4.2. LEMMA. There exist integers $0 \leq t_{0}<t_{1}<\cdots$, such that, if we set $E_{i}=[a, b]-\bigcup_{n=1}^{t_{i}}\left(a_{n}, b_{n}\right)$ for $i=0,1, \ldots$, then for each $i$,

1. $E_{i}$ can be written as $E_{i}=\bigcup_{j=1}^{c_{i}} I_{i j}$, where the $I_{i j}$ are pairwise disjoint nondegenerate closed arcs.

2. If $i \geq 1$ and if $1 \leq m \leq c_{i-1}$, then $E_{i} \cap I_{i-1 m}$ is the union of at least $N_{i}=r_{i 1}+r_{i 2}+\cdots+r_{i m_{i}}$ of the arcs $I_{i j}$ (where the positive integers $r_{i j}$ are those found in Lemma 3.5).

Proof. Clearly $E_{0}=[a, b]$ satisfies the conditions of the lemma. Assume that $t_{0}, t_{1}, \ldots, t_{s-1}$ have been chosen so that $t_{0} \leq t_{1}<\cdots<t_{s-1}$ and so that $E_{0}$, $E_{1}, \ldots, E_{s-1}$ satisfy conditions 1 and 2 .

Note first that each arc $I_{s-1 m}\left(1 \leq m \leq c_{s-1}\right)$ contains infinitely many of the $\operatorname{arcs}\left(a_{n}, b_{n}\right)$. Otherwise, $E \cap I_{s-1 m}$ would contain either a nondegenerate arc or an isolated point. If $E \cap I_{s-1 m}$ contains a nondegenerate arc, then so does $E$, contradicting the fact that $E$ is nowhere dense. If $E \cap I_{s-1 m}$ contains an isolated point, then so does $E$, since $I_{s-1 m}$ is separated from the other $\operatorname{arcs} I_{s-1 j}$ by open $\operatorname{arcs}$ of $[a, b]-E$. However, in the statement of Theorem $4.1 E$ is assumed to be perfect.

Since each of the $\operatorname{arcs} I_{s-1 m}$ contains infinitely many of the $\operatorname{arcs}\left(a_{n}, b_{n}\right)$, if we choose $t_{s}$ large enough we will have $t_{s-1}<t_{s}$ and, if $1 \leq m \leq c_{s-1}$, then $\bigcup_{n=1}^{t_{s}}\left(a_{n}, b_{n}\right)$ will contain at least $N_{s}-1$ of the arcs $\left(a_{n}, b_{n}\right)$ which lie in $I_{s-1 m}$. 
Let $E_{s}=[a, b]-\bigcup_{n=1}^{t_{s}}\left(a_{n}, b_{n}\right)$. Then $E_{s}$ can be written as $E_{s}=\bigcup_{j=1}^{c_{s}} I_{s j}$, where the $I_{s j}$ are pairwise disjoint closed subarcs of $[a, b]$. Note that the arcs $I_{s j}$ found at this step are nondegenerate since $E$ contains no isolated points. Since at least $N_{s}-1$ of the arcs $\left(a_{n}, b_{n}\right)$ were resolved from each arc $I_{s-1 m}$, at least $N_{s}$ of the $\operatorname{arcs} I_{s j}$ lie in $I_{s-1} m$. The lemma now follows by induction.

It follows from the definition of the sets $E_{i}$ in Lemma 4.2 that $E_{0} \supset E_{1} \supset \cdots$ and that $\bigcap_{i=0}^{\infty} E_{i}=E$. Let $F=\left\{I_{i j} \mid i=1,2, \ldots ; j=1,2, \ldots, c_{i}\right\}$, where the arcs $I_{i j}$ are those appearing in the statement of Lemma 4.2. Let $S=\bigcup_{i=1}^{\infty}\left(\left[q_{i-1}, q_{i}\right] \times E_{i}\right)$, where $\left\{q_{i}\right\}$ is the $R$-sequence appearing in the statement of Theorem 4.1. Choose a sequence of real numbers $\left\{r_{i}\right\}, i=1,2, \ldots$, such that, for each $i, q_{i-1}<r_{i}<q_{i}$. Recall Definition 3.9.

4.3. LEMMA. There exists a correspondence $\phi$ which associates to every arc $I_{i j}\left(i=1,2, \ldots ; j=1,2, \ldots, c_{i}\right)$ of $F$ a pair $\left(L_{i}, p_{i}\right)$, where $L_{i}$ is a continuum of level $i$ and $p_{i}$ is a bridge point on $L_{i}$, and there exists a continuous function $G(x, \tau): S \rightarrow \Omega$, where $\Omega$ is the finite plane. The correspondence $\phi$ and the function $G$ have the following properties:

1. If $\left\{\left(L_{i}, p_{i}\right)\right\}$ is any sequence of the form $*$, there exists a corresponding sequence $\left\{I_{i j_{i}}\right\}$ of arcs of $F$ such that $I_{1 j_{1}} \supset I_{2 j_{2}} \supset \cdots$ and such that $\phi\left(I_{i j_{i}}\right)=\left(L_{i}, p_{i}\right)$ for every positive integer $i$.

2. Suppose that $\left\{\left(L_{i}, p_{i}\right)\right\}$ is a sequence of the form * and that $\left\{I_{i j_{i}}\right\}$ is the corresponding nested sequence of arcs of $F$.

a. For each point $\tau \in I_{1 j_{1}}$,

(1) $G\left(\left[q_{0}, q_{1}\right] \times\{\tau\}\right)=L_{1}$.

(2) $G\left(q_{0}, \tau\right)=G\left(q_{1}, \tau\right)=p_{1}$.

b. For $i=2,3, \ldots$ we have, for each point $\tau \in I_{i j_{i}}$,

(1) $G\left(\left[q_{i-1}, r_{i}\right] \times\{\tau\}\right)$ is a direct path from $p_{i-1}$ to $p_{i}$.

(2) $G\left(\left[r_{i}, q_{i}\right] \times\{\tau\}\right)=L_{i}$.

(3) $G\left(q_{i-1}, \tau\right)=p_{i-1}$.

(4) $G\left(r_{i}, \tau\right)=G\left(q_{i}, \tau\right)=p_{i}$.

3. If $\left\{I_{i j_{i}}\right\}, i=1,2, \ldots$, is any nested sequence of arcs of $F$, then $\left\{\phi I_{i j_{i}}\right\}$ is some sequence $\left\{\left(L_{i}, p_{i}\right)\right\}$ of the form * with respect to which properties a and $\mathrm{b}$ of part 2 hold.

PROOF. The proof is somewhat lengthy, and we will divide it into several parts.

Part 1. There exists a correspondence $\phi$ which associates to each arc $I_{1 j}$ a pair of the form $\left(L_{1}, p_{1}\right)$, where $L_{1}$ is a continuum of level 1 and $p_{1}$ is a bridge point on $L_{1}$, and there exists a function $G(x, \tau)$, continuous and finite-valued on $\left[q_{0}, q_{1}\right] \times E_{1}$, with the following properties:

1. Each pair of the form $\left(L_{1}, p_{1}\right)$ is the image under $\phi$ of some arc $I_{1 j}, 1 \leq j \leq c_{1}$.

2. If $\phi\left(I_{1 j}\right)=\left(L_{1}, p_{1}\right)$, then for each point $\tau \in I_{1 j}$,

a. $G\left(\left[q_{0}, q_{1}\right] \times\{\tau\}\right)=L_{1}$.

b. $G\left(q_{0}, \tau\right)=G\left(q_{1}, \tau\right)=p_{1}$.

PROOF. Since there are $m_{1}$ continua $L_{1 j}$ of level 1 , and since there are $r_{1 j}$ bridge points $p_{1 j k}$ on each continuum $L_{1 j}$, there are $N_{1}=r_{11}+r_{12}+\cdots+r_{1 m_{1}}$ possible pairs of the form $\left(L_{1}, p_{1}\right)$. It follows from condition 2 of Lemma 4.2 that $E_{1}$ is the union of at least $N_{1}$ nondegenerate pairwise disjoint closed arcs $I_{1 j}$. Define the correspondence $\phi$ so that each pair $\left(L_{1}, p_{1}\right)$ is the image of at least one of the 
$\operatorname{arcs} I_{1 j}$. Suppose that $\phi\left(I_{1 j}\right)=\left(L_{1}, p_{1}\right)$. Since $L_{1}$ is a finite locally connected continuum, there exists a continuous finite-valued function $h$ mapping the interval $\left[q_{0}, q_{1}\right]$ onto $L_{1}$ with $h\left(q_{0}\right)=h\left(q_{1}\right)=p_{1}$. Define the continuous function $G$ by letting $G(x, \tau)=h(x)$ for each $\tau \in I_{1 j}$.

Since the intervals $I_{1 j}$ are pairwise disjoint closed intervals, the function $G$ is continuous on all of $\left[q_{0}, q_{1}\right] \times E_{1}$.

Part 2. For $i=1,2, \ldots, m$, suppose that the correspondence $\phi$ has been defined on each of the $\operatorname{arcs} I_{i j}, 1 \leq j \leq c_{i}$. Suppose, moreover, that the continuous function $G$ has been defined on $\bigcup_{i=1}^{m}\left[q_{i-1}, q_{i}\right] \times E_{i}$ and that, if $\phi\left(I_{m j}\right)=\left(L_{m}, p_{m}\right)$, then $G\left(q_{m}, \tau\right)=p_{m}$ for each $\tau \in I_{m j}$. Then, we can extend $\phi$ to the subarcs $I_{m+1 s}$, $1 \leq s \leq c_{m+1}$, of $E_{m+1}$ and we can extend $G$ continuously to $\left[q_{m}, q_{m+1}\right] \times E_{m+1}$ so that the following hold:

1. For each subarc $I_{m+1 s}$ of $E_{m+1}, \phi\left(I_{m+1 s}\right)$ is a pair of the form $\left(L_{m+1}, p_{m+1}\right)$.

2. Suppose that $I_{m j}$ is a subarc of $E_{m}$ and that $\phi\left(I_{m j}\right)=\left(L_{m}, p_{m}\right)$. Assume that $\left(L_{m+1}, p_{m+1}\right)$ is any pair for which

a. $L_{m+1}$ is a continuum of level $m+1$.

b. $p_{m+1}$ is a bridge point on $L_{m+1}$.

c. $d\left(L_{m}, L_{m+1}\right)<1 / 2^{m}$.

d. $d\left(p_{m}, p_{m+1}\right)<1 / 2^{m-1}$.

Then, there exists a subarc $I_{m+1 s}$ of $E_{m+1}$ with $I_{m+1 s} \subset I_{m j}$ and $\phi\left(I_{m+1 s}\right)=$ $\left(L_{m+1}, p_{m+1}\right)$.

3. Suppose that $I_{m j}$ and $I_{m+1 s}$ are subarcs of $E_{m}$ and $E_{m+1}$ respectively with $I_{m+1 s} \subset I_{m j}, \phi\left(I_{m j}\right)=\left(L_{m}, p_{m}\right)$ and $\phi\left(I_{m+1 s}\right)=\left(L_{m+1}, p_{m+1}\right)$. Then for all elements $\tau \in I_{m+1 s}$,

a. $G\left(\left[q_{m}, r_{m}\right] \times\{\tau\}\right)$ is a direct path from $p_{m}$ to $p_{m+1}$.

b. $G\left(\left[r_{m}, q_{m+1}\right] \times\{\tau\}\right)=L_{m+1}$.

c. $G\left(q_{m}, \tau\right)=p_{m}$.

d. $G\left(r_{m}, \tau\right)=G\left(q_{m+1}, \tau\right)=p_{m+1}$.

4. If the $\operatorname{arcs} I_{m j}$ and $I_{m+1 s}$ satisfy the hypotheses of part 3, then

$$
d\left(L_{m}, L_{m+1}\right)<1 / 2^{m}
$$

and

$$
d\left(p_{m}, p_{m+1}\right)<1 / 2^{m-1} .
$$

PROOF. Let the subarc $I_{m j}$ of $E_{m}$ be given and suppose that $\phi\left(I_{m j}\right)=\left(L_{m}, p_{m}\right)$. Form all pairs $\left(L_{m+1}, p_{m+1}\right)$ having properties a-d of part 2 . There are at most $N_{m+1}$ such pairs. By Lemma $4.2, E_{m+1} \cap I_{m j}$ is the union of at least $N_{m+1}$ of the subarcs $I_{m+1 s}$ of $E_{m+1}$. Thus, for each arc $I_{m+1 s} \subset I_{m j}$, we can insure that $\phi\left(I_{m+1 s}\right)$ is a pair of the form $\left(L_{m+1}, p_{m+1}\right)$ and that each such pair is the image of at least one subarc $I_{m+1 s} \subset I_{m j}$. If $\phi\left(I_{m+1 s}\right)=\left(L_{m+1}, p_{m+1}\right)$, define $G$ on $\left[q_{m}, q_{m+1}\right] \times I_{m+1 s}$ so that $G$ is continuous and satisfies conditions a-d of part 3 . This can be done as in the proof of part 1 of the lemma.

Repeat the above construction for each subarc $I_{m j}$ of $E_{m}$. Since distinct subarcs $I_{m+1 s}$ of $E_{m+1}$ are disjoint closed sets, $G$ has been extended continuously to all of $\left[q_{m}, q_{m+1}\right] \times E_{m+1}$. It is straightforward to verify that conditions 1-4 are satisfied. Lemma 4.3 now follows by induction.

Since $E_{i} \supset E$ for each $i$, we may restrict the function $G$ to $\left[q_{0}, R\right) \times E$. 
4.4. LEMMA. Suppose that $\left\{\left(L_{i}, p_{i}\right)\right\}$ and $\left\{\left(L_{i}^{\prime}, p_{i}^{\prime}\right)\right\}$ are distinct sequences of the form * and that $\left\{I_{i j_{i}}\right\}$ and $\left\{I_{i j_{i}}^{\prime}\right\}$ are nested sequences of arcs of $F$ with $\phi\left(I_{i j_{i}}\right)=\left(L_{i}, p_{i}\right)$ and $\phi\left(I_{i j_{i}}^{\prime}\right)=\left(L_{i}^{\prime}, p_{i}^{\prime}\right)$ for each $i$. Then

1. $\bigcap_{i=1}^{\infty} I_{i j_{i}}$ is some point $\tau \in E, \bigcap_{i=1}^{\infty} I_{i j_{i}}^{\prime}$ is some point $\tau^{\prime} \in E$ and

2. $\tau \neq \tau^{\prime}$.

Proof. For each $i, I_{i j_{i}} \subset E_{i}$. Since $\bigcap_{i=1}^{\infty} E_{i}=E, \bigcap_{i=1}^{\infty} I_{i j_{i}}$ is a subset of $E$. Since the $I_{i j_{i}}$ form a nested sequence of closed arcs, the intersection is nonempty. Since $E$ is nowhere dense, the intersection is some point $\tau \in E$. Similarly, $\bigcap_{i=1}^{\infty} I_{i j_{i}}^{\prime}$ is some point $\tau^{\prime} \in E$.

Since $\left\{\left(L_{i}, p_{i}\right)\right\}$ and $\left\{\left(L_{i}^{\prime}, p_{i}^{\prime}\right)\right\}$ are distinct sequences of the form $*$, for some $k$, $\left(L_{k}, p_{k}\right) \neq\left(L_{k}^{\prime}, p_{k}^{\prime}\right)$. But then it follows from the construction of $E_{k}$ that $I_{k j_{k}}$ and $I_{k j_{k}}^{\prime}$ are disjoint so that $\tau \neq \tau^{\prime}$.

Let us denote by $C_{\tau}(G)$ the cluster set of $G(x, \tau)$ as $x \rightarrow R$.

4.5. Lemma. Let the continuum $K \in \mathcal{L}$ be given. Then there exist $2^{\aleph_{0}}$ points $\tau \in E$ for which $C_{\tau}(G)=K$.

PROOF. Choose some point $p \in K$. It follows from Lemma 3.11 that there are $2^{\aleph_{0}}$ sequences $\left\{\left(L_{i}, p_{i}\right)\right\}$ of the form $*$ with $\lim _{i \rightarrow \infty} L_{i}=K$ and $\lim _{i \rightarrow \infty} p_{i}=p$. Let $\left\{\left(L_{i}, p_{i}\right)\right\}$ be one such sequence. It follows from condition 1 of Lemma 4.3 that there is a nested sequence $\left\{I_{i j_{i}}\right\}$ of arcs of $F$ with $\phi\left(I_{i j_{i}}\right)=\left(L_{i}, p_{i}\right)$ for each $i$. From Lemma 4.4, $\bigcap_{i=1}^{\infty} I_{i j_{i}}$ is some point $\tau \in E$.

We must establish that $C_{\tau}(G)=K$.

Suppose first that $w$ is a point of $K$. Since $\lim _{i \rightarrow \infty} L_{i}=K$, there exist points $w_{i} \in L_{i}$ for each $i$ with $\lim _{i \rightarrow \infty} d\left(w_{i}, w\right)=0[\mathbf{9}$, p. 335; 10, p. 49]. But then, by condition 2 of Lemma 4.3, there exist points $x_{i} \in\left[q_{i-1}, q_{i}\right]$ with $G\left(x_{i}, \tau\right)=w_{i}$ so that $w \in C_{\tau}(G)$. Suppose now that $w \in C_{\tau}(G)$. There exists a sequence $\left\{x_{n}\right\}$ of points of $\left[q_{0}, R\right)$ such that $\lim _{n \rightarrow \infty} x_{n}=R$ and $\lim _{n \rightarrow \infty} G\left(x_{n}, \tau\right)=w$. By considering subsequences we may assume that either

1. for each $n, x_{n}$ is a point of $\left[r_{i_{n}}, q_{i_{n}}\right]$ where $\lim _{n \rightarrow \infty} i_{n}=\infty$ or

2. for each $n, x_{n}$ is a point of $\left[q_{i_{n}-1}, r_{i_{n}}\right]$ where $\lim _{n \rightarrow \infty} i_{n}=\infty$.

In case 1 , by condition 2 of Lemma $4.3, G\left(x_{n}, \tau\right)$ is a point of the continuum $L_{i_{n}}$ for each $n$. Since the sequence $\left\{\left(L_{i}, p_{i}\right)\right\}$ was chosen so that $\lim _{i \rightarrow \infty} L_{i}=K$, $\lim _{n \rightarrow \infty} L_{i_{n}}=K$ and $w=\lim _{n \rightarrow \infty} G\left(x_{n}, \tau\right)$ is some point of $K[\mathbf{9}$, p. 339; 10, p. 49].

In case 2, again by condition 2 of Lemma $4.3, G\left(x_{n}, \tau\right)$ lies on a direct path from $p_{i_{n}-1}$ to $p_{i_{n}}$ for each $n$. But the sequence $\left\{\left(L_{i}, p_{i}\right)\right\}$ was chosen so that $\lim _{i \rightarrow \infty} p_{i}=p$, where $p$ is the point we selected on $K$. But then, $\lim _{n \rightarrow \infty} p_{i_{n}}=p$. By Definition 3.8, $w=\lim _{n \rightarrow \infty} G\left(x_{n}, \tau\right)=p \in K$.

Thus, in either case, $w \in K$. Since $w$ was an arbitrary point of $C_{\tau}(G), C_{\tau}(G) \subseteq$ $K$. Thus, finally, $C_{\tau}(G)=K$.

It now follows from Lemma 4.4 and the previous discussion that there exist $2^{\aleph_{0}}$ points $\tau \in E$ for which $C_{\tau}(G)=K$.

Essentially, the previous sequence of lemmas insures that every continuum $K \in \mathcal{L}$ appears as the cluster set of $G$ at sufficiently many $\tau$ levels. We now consider uniformity of covergence. 
Since the function $G$ is continuous, $C_{\tau}(G)$ is a continuum in the space $\mathcal{L}$ for each $\tau \in E$. Moreover, as $i$ increases, the sets $\overline{G\left(\left[q_{i}, R\right) \times\{\tau\}\right)}$ form a nested sequence of continua with $C_{\tau}(G)=\bigcap_{i=1}^{\infty} \overline{G\left(\left[q_{i}, R\right) \times\{\tau\}\right)}$.

4.6. LEMMA. If $\tau \in E$ and if $x \in\left[q_{n}, R\right)$, then there exists a point $b \in C_{\tau}(G)$ for which $d(G(x, \tau), b)<1 / 2^{n-2}$.

ProOF. It follows from the construction of the sets $E_{i}$ that there exists a nested sequence $\left\{I_{i j_{i}}\right\}$ of arcs of $F$ with $\{\tau\}=\bigcap_{i=1}^{\infty} I_{i j_{i}}$. By condition 3 of Lemma 4.3, there exists a sequence $\left\{\left(L_{i}, p_{i}\right)\right\}$ of the form $*$ with $\varphi\left(I_{i j_{i}}\right)=\left(L_{i}, p_{i}\right)$ for each $i$. By Lemma $3.10 \lim _{i \rightarrow \infty} L_{i}$ exists and is some continuum $K \in \mathcal{L}$, and $\lim _{i \rightarrow \infty} p_{i}$ exists and is some point $p \in K$. It then follows from condition 3 of Lemma 4.3 and the proof of Lemma 4.5 that $C_{\tau}(G)=K$.

Now, suppose that $x$ is some real number with $q_{n} \leq x<R$. We wish to find a point $b \in C_{\tau}(G)$ with $d(G(x, \tau), b)<1 / 2^{n-2}$. There are two cases.

Case 1. Suppose that for some integer $j>n, q_{j-1} \leq x \leq r_{j}$. Then, by condition 3 of Lemma $4.3, G(x, \tau)$ is a point on a direct path from $p_{j-1}$ to $p_{j}$. Since by Definition $3.9 d\left(p_{j-1}, p_{j}\right)<1 / 2^{j-2}$, it follows from Definition 3.8 that $d\left(G(x, \tau), p_{j}\right)<1 / 2^{j-2}$. By Lemma $3.10 d\left(p_{j}, p\right)<1 / 2^{j-2}$. Thus,

$$
d(G(x, \tau), p)<1 / 2^{j-3} .
$$

Take $b=p$.

Case 2. Suppose that for some integer $j>n, r_{j} \leq x \leq q_{j}$. Then by condition 3 of Lemma $4.3 G(x, \tau)$ is a point on the continuum $L_{j}$. By Lemma 3.10

$$
d\left(L_{j}, K\right)<1 / 2^{j-1} .
$$

But then, since $K$ is compact, it follows from the definition of the distance $d$ that there exists a point $b \in K$ with $d(G(x, \tau), b)<1 / 2^{j-1}$.

Thus, in either case, there is some point $b \in K$ with $d(G(x, \tau), b)<1 / 2^{n-2}$.

4.7. LEMMA. Let $\varepsilon>0$ be given. Then there exists an integer $n=n(\varepsilon)$ such that, if $i \geq n$ and $\tau \in E$, we have $d\left(\overline{\left.G\left[q_{i}, R\right) \times\{\tau\}\right)}, C_{\tau}(G)\right)<\varepsilon$.

ProOF. Choose an $n$ so that $1 / 2^{n-2}<\varepsilon$. If $i \geq n$, we noted that $C_{\tau}(G) \subseteq$ $\overline{G\left(\left[q_{i}, R\right) \times\{\tau\}\right)} \subseteq \overline{G\left(\left[q_{n}, R\right) \times\{\tau\}\right)}$. The proof is now a straightforward application of Lemma 4.6.

Finally, we are ready to construct the analytic function $f$ promised in Theorem 4.1.

Proof OF TheOREM 4.1. Let $T=\bigcup_{\tau \in E} t_{\tau} \cap\left(q_{0} \leq|z|<R\right)$. Define the function $g$ on $T$ by the requirement that $g(z)=G(|z|, \tau)$, where $\tau$ is the unique $\tau \in E$ such that $z \in t_{\tau}$. Since $G$ is continuous on $\left[q_{0}, R\right) \times E$ and since every tress is a restricted tress, $g$ is continuous on $T$ (see $[\mathbf{4}$, p. 197 and 13, p. 82]). The existence of the function $f$, analytic in $|z|<R$, now follows directly from Arakeljan's theorem on tangential approximation (see [1, p. 286 and 7, p. 461]). Since the rate of convergence of $f(z)$ to $g(z)$ for $z \in T$ can be governed by $|z|$ (independent of $\tau$ ) the verification that $f$ has properties 1 and 2 of Theorem 4.1 is a straightforward application of Lemmas 4.5 and 4.7 .

The technique of "approximating" a continuum by a locally connected continuum was employed by F. Bagemihl and W. Seidel in [4], and the technique of using a 
function of the form $G(|z|, \tau)$ to construct a continuous function $g(z)$ on $T$ was employed in $[\mathbf{3}]$.

5. Closed sets of continua. Suppose that $\mathcal{F}$ is any closed set of continua on the Riemann sphere. We may modify the construction used in the proof of Theorem 4.1 by choosing the continua of level $i$ to have not only the two properties of Lemma 3.5 , but also the following additional property:

3. Given any continuum $L_{i j}$ of level $i$, there is some continuum $K \in \mathcal{F}$ for which $d\left(K, L_{i j}\right)<1 / 2^{i+1}$.

The choice is possible since any closed subset $\mathcal{F}$ of continua is compact. The locally connected continua of level $i$ need not, of course, be elements of $\mathcal{F}$. However, if $\left\{\left(L_{i}, p_{i}\right)\right\}$ is any sequence of the form $*, \lim _{i \rightarrow \infty} L_{i}$ will exist as before and will be some continuum $K$ of the closed set $\mathcal{F}$. Thus, the method of construction used in the proof of Theorem 4.1 (modified as indicated above) may be used to prove the following theorem:

5.1. THEOREM. Let $R, 0<R \leq+\infty$, be given, and let $\left\{q_{i}\right\}$ be an $R$-sequence. Let $\mathcal{F}$ be any closed set of continua on the Riemann sphere. Suppose that $E$ is a perfect, nowhere dense set on the circle $|\tau|=1$ and that $\tau$ is a tress in $|z|<R$ with respect to $E$ such that it is possible to take $E_{n}=E$ for all $n$ in the definition of a tress. Then there exists a function $f$, analytic in $|z|<R$, with the following properties:

1. For every continuum $K \in \mathcal{F}$, there exist $2^{\aleph_{0}}$ mb-paths $t_{\tau}$ of $\tau$ for which $C_{t_{\tau}}(f)=K$.

2. For every mb-path $t_{\tau}$ of $\tau, C_{t_{\tau}}(f)$ is some continuum $K \in \mathcal{F}$.

3. Convergence to the cluster set is uniform for all $m b$-paths $t_{\tau}$ of $\tau$.

As an immediate consequence, we can construct a function $f$, analytic in $|z|<R$, with prescribed asymptotic values in the following sense:

5.2. COROLlary. Let $R, 0<R \leq+\infty$, be given, and let $\left\{q_{i}\right\}$ be an $R$ sequence. Let $F$ be any closed set of points on the Riemann sphere. Suppose that $E$ is a perfect, nowhere dense set on the circle $|\tau|=1$ and that $\tau$ is a tress in $|z|<R$ with respect to $E$ such that it is possible to take $E_{n}=E$ for all $n$ in the definition of a tress. Then there exists a function $f$, analytic in $|z|<R$, with the following properties:

1. For every point $w \in F$, there are $2^{\aleph_{0}}$ mb-paths $t_{\tau}$ of $\tau$ along which $f$ has the asymptotic value $w$.

2. If $t_{\tau}$ is any mb-path of $\tau$, then $f$ has an asymptotic value $w$ on $t_{\tau}$, and $w \in F$.

3. Convergence to the asymptotic value is uniform on all mb-paths of $\tau$.

ProOF. The mapping $w \rightarrow\{w\}$ takes $F$ to a closed set of continua in $2^{X}$.

The above corollary is similar to Theorem 1 of $[\mathbf{5}]$.

If we let the closed set $\mathcal{F}$ of continua take certain special forms, a number of similar corollaries follow. We mention a few brief examples.

Let $A$ be any subset of the Riemann sphere $X$, and let $₹$ be the collection of all subcontinua $K$ of $X$ for which $A \subseteq K$. Then, $\xi$ is closed in $2^{X}[\mathbf{9}$, p. 162; 10, p. 49]). In this case, the points of $A$ occur as cluster values on every mb-path of the tress $\tau$. Moreover, every possible cluster set $K$ containing these values occurs as $C_{t_{\tau}}(f)$ on $2^{\aleph_{0}}$ mb-paths of $\tau$. Theorem 4.1 corresponds to the case in which 
$A=\Phi$. If $A$ is not itself a continuum, none of the cluster sets will, of course, be exactly $A$. If $A$ is closed (so an element of $2^{X}$ ) but not connected, the cluster sets need not approach $A$ in the metric $d$. For instance, if $A=\{p, q\}$ with $d(p, q)=\varepsilon$, there is no continuum $K$ with $d(K, A)<\varepsilon / 2$.

The set of subcontinua $K$ of $X$ with $K \subseteq A$ is not closed. However, if $F$ is a nonempty closed subset of $X$, the collection $₹$ of those continua $K$ with $K \subseteq F$ is closed in $2^{X}$. Thus, for instance, an analytic function $f$ may be constructed so that all and only those continua $K$ which are subsets of some closed disk $D$ occur as cluster sets of $f$ on mb-paths of $\tau$. Theorem 5.1 corresponds to the case in which $D$ is the entire Riemann sphere $X$. Again, if the closed set $F$ is not itself a continuum, the cluster sets may remain bounded away from $F$ in the metric $d$.

As another example, let $D$ be any closed subset of the interval $[0,2]$, and let $₹$ be the collection of those continua $K$ on the Riemann sphere whose diameter is an element of $D$. Then $\mathcal{F}$ is closed in $2^{X}[\mathbf{1 0}, \mathrm{p}$. 35]. In particular, we may construct an analytic function $f$ in $|z|<R$ such that $C_{t_{\tau}}(f)$ has diameter, say, $1 / 2$ on every mb-path $t_{\tau}$ of the tress $\tau$. Theorem 4.1 corresponds to the case in which $D$ is the entire interval $[0,2]$.

The construction technique may be extended to the case of $F_{\sigma}$ sets of continua. Suppose that $K=\bigcup_{n=1}^{\infty} \mathcal{F}_{n}$, where each $\mathcal{F}_{n}$ is a closed subset of $\mathcal{L}$. Suppose that $\tau$ is a tress with respect to the set $E=\bigcup_{n=1}^{\infty} E_{n}$, where each $E_{n}$ is perfect and nowhere dense. Let $\left\{q_{i}\right\}$ be an $R$-sequence. For each $n$, let $T_{n}=\bigcup_{\tau \in E_{n}}\left(t_{\tau} \cap\left[q_{n}, R\right)\right)$. For each $n$, what is required is to construct the function $G(x, \tau)$ and the continuous function $g(z)$ corresponding to the closed set $\mathcal{F}_{n}$ on the paths of $T_{n}$. For convenience, we may use the $R$-sequence $\left\{q_{i n}\right\}$, where $q_{i n}=q_{i+n}$ for each $i$. Convergence to the cluster set is no longer uniform. Thus, for $F_{\sigma}$ sets of continua we have the following theorem.

5.3. THEOREM. Let $R, 0<R \leq+\infty$, be given. Suppose that $E=\bigcup_{n=1}^{\infty} E_{n}$, where each $E_{n}$ is a perfect, nowhere dense set on the circle $|\tau|=1$ and that $\bar{T}$ is a tress in $|z|<R$ with respect to $E$. Let $\mathcal{K}$ be any $F_{\sigma}$ set of continua on the Riemann sphere. Then there exists a function $f$, analytic in $|z|<R$, with the following properties: $K$.

1. For every continuum $K \in \mathcal{K}$, there exist $2^{\aleph_{0}}$ mb-paths $t_{\tau}$ of $T$ with $C_{t_{\tau}}(f)=$

2. If $t_{\tau}$ is any $m b$-path of $\tau$, then $C_{t_{\tau}}(f)$ is some continuum $K \in \mathcal{K}$.

6. Weaker approximation theorems. It should be noted that the above results can be obtained using the earlier approximation theorem of S. N. Mergelyan $[\mathbf{1 1}]$.

In fact, similar results can be derived using even earlier interpolation and approximation theorems of J. L. Walsh [14]. Substantial control over the form of the mb-paths is still possible. In particular, the paths may be required to all lie in some central angle, to all be spirals, or to all end at some point on $|z|=R$. They may not, however, be required to be radii. The topological machinery remains unchanged. The tress of our previous discussion is replaced by a particularly simple type of path family tailored to Walsh's theorems.

We content ourselves with introducing the path family, sketching the construction of the continuous function $g$, and stating the main result. 
Recall that, if $\left\{q_{i}\right\}$ is a $R$-sequence, $A_{i}$ denotes the closed annulus $q_{i-1} \leq|z|<q_{i}$. Let $C_{i}$ denote the circle $|z|=q_{i}$.

6.1. Definition. Suppose that $0<R \leq+\infty$. Let $\left\{q_{i}\right\}$ be an $R$-sequence, and let $\left\{N_{i}\right\}, i=1,2, \ldots$, be a sequence of positive integers. If $T$ is a subset of $A_{1} \cup A_{2} \cup \cdots$, then $T$ is called a tree with respect to the sequences $\left\{q_{i}\right\}$ and $\left\{N_{i}\right\}$ if $T$ satisfies the following conditions:

1. $T \cap A_{1}$ is a union of $N_{1}$ disjoint closed line segments, each extending from $C_{0}$ to $C_{1}$.

2. For $i=1,2, \ldots, T \cap A_{i+1}$ is a union of $M_{i+1}=N_{1} \cdot N_{2} \cdot \ldots \cdot N_{i+1}$ closed line segments, $S_{1}, S_{2}, \ldots, S_{M_{i+1}}$, each extending from $C_{i}$ to $C_{i+1}$. Moreover, the segments have the following properties:

a. If $1 \leq j \leq M_{i+1}$, there exists a line segment $S$, lying in $T$ and extending from $C_{i-1}$ to $C_{i}$, for which $S \cap C_{i}=S_{j} \cap C_{i}$.

b. If $1 \leq j \leq M_{i+1}$ and $1 \leq k \leq M_{i+1}$, and if $j \neq k$, then the line segments $S_{j}$ and $S_{k}$ are either disjoint or intersect only at a point of $C_{i}$.

c. If $S$ is a segment lying in $T$ and extending from $C_{i-1}$ to $C_{i}$, then there are $N_{i+1}$ of the segments $S_{j}, 1 \leq j \leq M_{i+1}$, for which $S \cap C_{i}=S_{j} \cap C_{i}$.

If $N_{1}>1$, then a tree is not a connected set. It is not essential that $T \cap A_{i}$ be composed of line segments. The line segments may be replaced by Jordan arcs extending montonically from $C_{i-1}$ to $C_{i}$.

The path family of interest consists of mb-paths $t$ which lie as point sets in an appropriate tree $T$. The tree is to be constructed with respect to some $R$-sequence $\left\{q_{i}\right\}$ and the sequence of integers $\left\{N_{i}\right\}$, where $N_{i}=r_{i 1}+r_{i 2}+\cdots+r_{i m_{i}}$, and the integers $r_{i j}$ are those found in Lemma 3.5. Choose a sequence $\left\{r_{i}\right\}, i=1,2, \ldots$, such that, for each $i, q_{i-1}<r_{i}<q_{i}$. For each $i$, let $C_{i}^{\prime}$ denote the circle $|z|=r_{i}$. Also, let $A_{i}^{\prime}$ denote the closed annulus $q_{i-1} \leq|z| \leq r_{i}$, and let $A_{i}^{\prime \prime}$ denote the annulus $r_{i} \leq|z| \leq q_{i}$.

If $S$ is a line segment of $T$ extending from $C_{i-1}$ to $C_{i}$, then $S \cap C_{i-1}$ will be called the initial point of $S$ and $S \cap C_{i}$ will be called the terminal point. If $\left\{S_{i}\right\}$, $i=1,2, \ldots$, is a sequence of line segments of $T$, where each segment $S_{i}$ extends from $C_{i-1}$ to $C_{i}$, then the sequence will be called a sequence of consecutive line segments in $T$ if, for each $i$, the terminal point of $S_{i}$ is the initial point of $S_{i+1}$.

The basic construction lemma for the continuous function $g$ is the following. The proof parallels the proof of Lemma 4.3.

6.2. Lemma. Let $T$ be a tree as described above. Then there exists a continuous finite valued function $g: T \rightarrow X$ with the following properties:

1. If $\left\{\left(L_{i}, p_{i}\right)\right\}$ is any sequence of the form *, there exists a sequence $\left\{S_{i}\right\}$ of consecutive line segments lying in $T$ such that

a. (1) $g\left(S_{1}\right)=L_{1}$.

(2) $g\left(S_{1} \cap C_{0}\right)=g\left(S_{1} \cap C_{1}\right)=p_{1}$.

b. For $i=1,2, \ldots$,

(1) $g\left(S_{i} \cap A_{i}^{\prime}\right)$ is a direct path from $p_{i-1}$ to $p_{i}$.

(2) $g\left(S_{i} \cap A_{i}^{\prime \prime}\right)=L_{i}$.

(3) $g\left(S_{i} \cap C_{i-1}\right)=p_{i-1}$.

(4) $g\left(S_{i} \cap C_{i}^{\prime}\right)=g\left(S_{i} \cap C_{i}\right)=p_{i}$. 
2. Moreover, if $\left\{S_{i}\right\}, i=1,2, \ldots$, is any sequence of consecutive line segments lying in $T$, there is some sequence $\left\{\left(L_{i}, p_{i}\right)\right\}$ of the form * with respect to which the sequence $\left\{S_{i}\right\}$ has properties a and $\mathrm{b}$ above.

We may now use the approximation technique of F. Bagemihl and W. Seidel to construct the analytic function $f$ (see [5, pp. 1251-1254 and 4, pp. 187-190]). The polynomial interpolation and approximation theorems required are those of $\mathrm{J}$. L. Walsh (see [14, pp. 47, 310]). We are thus lead to the following theorem.

6.3. THEOREM. Let $R, 0<R \leq+\infty$, be given, and let $\left\{q_{i}\right\}$ be an $R$-sequence. Then there exists a tree $T$ in $|z|<R$ with respect to the $R$-sequence $\left\{q_{i}\right\}$, and there exists a function $f$, analytic in $|z|<R$, with the following properties:

1. If $K$ is any continuum on the Riemann sphere, there exist $2^{\aleph_{0}}$ mb-paths $t$, lying in $T$, for which $C_{t}(f)=K$.

2. Convergence to the cluster set is uniform for all mb-paths $t$ lying in $T$.

The construction technique for a tree, outlined above, leads directly to corollaries which parallel those of $\S 5$.

ACKNOWLEDGMENTS. I would like to thank the referee for helpful comments and suggestions.

The basic construction formed part of my dissertation under the late Professor W. Seidel. His guidance is gratefully remembered. Revision and extension of the original work was made possible by a Rackham Faculty Fellowship from the University of Michigan.

\section{REFERENCES}

1. N. U. Arakeljan, Uniform and tangential approximation by holomorphic functions, Izv. Akad. Nauk. Armjan SSR Ser. Mat. 3 (1968), 273-286.

2. F. Bagemihl and J. E. McMillan, Uniform approach to cluster sets of arbitrary functions in a disk, Acta Math. Acad. Sci. Hungar. 17 (1966), 411-418.

3. F. Bagemihl and W. Seidel, A problem concerning the cluster sets of analytic functions, Math. Z. 62 (1955), 99-110.

4. __ Some boundary properties of analytic functions, Math. Z. 61 (1954), 186-199.

5. S__ Spiral and other asymptotic paths, and paths of complete indetermination of analytic and meromorphic functions, Proc. Nat. Acad. Sci. U.S.A. 39 (1953), 1251-1258.

6. J. Dugundji, Topology, Allyn and Bacon, Boston, Mass., 1966.

7. P. M. Gauthier and W. Seidel, Some applications of Arakelian's approximation theorems to the theory of cluster sets, Izv. Akad. Nauk Armjan SSR Ser. Mat. 6 (1971), 458-464.

8. S. Kierst and E. Szpilrajn, Sur certaines singularités des fonctions analytiques uniformes, Fund. Math. 21 (1933), 276-294.

9. K. Kuratowski, Topology. I, Academic Press, New York, 1966.

10. Topology. II, Academic Press, New York, 1968.

11. S. N. Mergelyan, Uniform approximation to functions of a complex variable, Uspekhi Mat. Nauk (N.S.) 7 (1952), 33-122; Amer. Math. Soc. Transl. 101 (1954).

12. A. Roth, Appoximationseigenschaften und strahlengrenzewerte Meromorpher und ganzer Funktionen, Comment. Math. Helv. 11 (1938), 77-125.

13. J. L. Stebbins, A construction of meromorphic functions with prescribed boundary behavior, Nagoya Math. J. 41 (1971), 75-87.

14. J. L. Walsh, Interpolation and approximation by rational functions in the complex domain, Amer. Math. Soc. Colloq. Publ., vol. 20, Amer. Math. Soc., Providence, R.I., 1960. 